\title{
What will make patients use a patient decision aid? a qualitative study on patients' perspectives on implementation barriers and facilitators
}

\begin{abstract}
Rationale, aims, and objectives: Few studies focus on patients' views on factors influencing implementation of patient decision aids (PDAs). This study aims to explore patients' views on the factors influencing implementation of an "insulin choice" PDA in a primary care setting. Methods: This study used a descriptive qualitative study design. Interviews were conducted using a semistructured interview guide developed based on the theoretical domains framework. Nine in-depth interviews and three focus group discussions were conducted with patients with type 2 diabetes who have been advised to start insulin or were currently using insulin and those who had been seeking diabetes treatment in the clinic for more than 1 year. Interviews were conducted after the participants were familiarized with the PDA. Data were analysed using a thematic approach. Results: Five themes emerged from the data analysis: (a) trust in the physician (patients preferred physicians to other health care providers in delivering the insulin PDA to them as they trusted physicians more when it comes to making decisions such as starting insulin), (b) physician's attitude (patients were more likely to trust a physician who is friendly and sympathetic hence would be more willing to use the insulin PDA), (c) physician's communication style (patients were more willing to use the insulin PDA if the physicians would take time and guide them in the PDA use), (d) conducive environment (patients preferred to read the PDA at home), and (e) cost (patients would not be willing to pay to use the insulin PDA unless they needed it). Conclusions: Patients want physicians to play a major role in the implementation of the insulin PDA; physicians' communication style and commitment may influence implementation outcomes. Health care authorities need to create a conducive environment and provide patients with free access to PDA to promote effective implementation.
\end{abstract}

Keyword: Barriers; Decision support; facilitators; Implementation; Insulin initiation; Patient decision aid; Patients' perspectives; Type 2 diabetes mellitus 